\title{
Asimilación del Almacén de Datos en las Organizaciones Corporativas Petroquímicas
}

\author{
Patricia Silva ${ }^{1,2}$ y Rebeca Silva ${ }^{1}$ \\ (1) Instituto Tecnológico de Ciudad Madero, División de Estudios de Posgrado, Av. $1^{\circ}$ de Mayo esq. \\ Sor Juana Inés de la Cruz s/n, Col. Los Mangos, 89440 Cd. Madero, Tamaulipas-México \\ (2) Universidad Autónoma de Tamaulipas, FCAT, Centro Universitario Tampico - Madero s/n, \\ Tampico, Tamaulipas-México (e-mail: patsil@prodigy.net.mx)
}

\begin{abstract}
Resumen
El objetivo del trabajo que se presenta fue analizar el proceso de asimilación del almacén de datos en las organizaciones corporativas petroquímicas en el estado de Tamaulipas-México. Se evaluó el efecto del grado de adopción de la tecnología por el capital humano de la organización, y en la toma de decisiones. El proceso de asimilación del almacén de datos en el caso estudiado, disminuye el tiempo, traduciéndose en un incremento de la exactitud y precisión para la toma de decisiones. Esto se refleja en un $93.9 \%$ en la rentabilidad. Sin embargo, el estudio muestra también que no se explotan al máximo las potencialidades del almacén de datos.
\end{abstract}

Palabras clave: almacén de datos, arquitectura de información, organizaciones corporativas, rentabilidad

\section{Data Warehouse Assimilation in the Petrochemical Corporative Organizations}

\begin{abstract}
The purpose of this work was to analyze the data warehouse assimilation process in the petrochemical corporative organizations in the state of Tamaulipas-Mexico. The effect of the adoption degree among the personnel, and in making decisions was evaluated. The process of adoption of the data warehouse in the case studied in this work reduces time, which causes an increase in the accuracy and precision of decision making. This is shown by the $93.9 \%$ profit. However, the study also shows that the maximum potentialities of the data warehouse are not fully used.
\end{abstract}

Keywords: data warehouse, architecture information, corporative organizations, profit 


\section{INTRODUCCIÓN}

Con la llegada del Terabyte (1000Gb) en los noventas, se creó el almacén de datos y en el año 2002 llega el Petabyte (1024 Terabytes) como el equivalente de doscientos cincuenta mil millones páginas de texto (Pérez, 2006). Algunos autores definen el almacén de datos como un administrador de base de datos (Giovinazzo e Inmon, 2001), como una arquitectura de datos (Koncilia, 2007) y otros como una fuente de datos comprimidos e integrados (Lee et al., 2006). Para fines de este trabajo se define como una arquitectura de información con diversos componentes siendo el más importante la minería de datos que le proporciona cierto nivel de inteligencia, con un enfoque sumarizado e histórico en los datos, que sea un soporte técnico en el proceso de toma de decisiones en los niveles táctico, estratégico y operacional en una organización corporativa petroquímica. Lo que se ha hecho antes sirve como base en la realización del análisis de asimilación del almacén de datos, fue seleccionado por estar enfocado a la tecnología de información del almacén de datos, estar fundamentado en los modelos de etapas de Cota (2007), complementándose con otros modelos ya probados (Mundy et al., 2006), el modelo propuesto es mejor porque se considera que los objetivos de la organización estén alineados al almacén de datos, estableciendo métricas para evaluarlo antes y después de adquirilo (Gómez, 2006), basándose en la teoría de la medida, cuyo objetivo es obtener la escala matemática a la que pertenece una métrica y especificar un marco general en el que las medidas deben ser definidas (Serrano, 2008), fundamentándose en un plan informático para un entorno cambiante, siendo una característica de un almacén de datos exitoso, permitiendo la expansión de la tecnología en la organización (Sturm, 2006), capacitación, se considera la intervención de agentes de cambio y de expertos, porque ninguna de las aplicaciones de almacén de datos por muy sofisticada que sea, aporta ventajas competitivas sostenibles por si mismas, la ventaja se obtiene en el desarrollo en el uso, principalmente en funcionalidad y establecimiento de métricas.

El almacén de datos cumple con el papel fundamental de dar confianza a los procesos de las organizaciones corporativas (Malori et al., 2003). El problema que se aborda es la falta de conocimientos tecnológicos para la adopción de la tecnología y al respecto el que adquiere la tecnología, manifiesta un desencanto hacia el almacén de datos, debido a que se crearon falsas expectativas en el sentido de que las tecnologías solucionan todos los problemas de su organización.

Se concibe por grado de adopción a la velocidad relativa con la cual una innovación es adoptada por el capital humano de un sistema social. Las organizaciones corporativas petroquímicas adquieren el almacén de datos, sin entender su funcionamiento y se enfrentan al problema de la falta de asimilación tecnológica, lo que ocasiona que las tecnologías y sistemas que existen no hayan sido explotadas eficientemente debido a la evolución natural del procesamiento de datos.

El objetivo general es analizar el proceso de asimilación del almacén de datos en la toma de decisiones y su efecto en la rentabilidad de las organizaciones corporativas petroquímicas en el estado de Tamaulipas, México. Con una inversión que es superior a los cinco mil quinientos millones de dólares (Hernández, 2005), en donde el negocio petroquímico es una de las principales actividades económicas del país, su mercado es de los más grandes, ya que se podría estimar que en el estado se produce el $30 \%$ de los petroquímicos del país, si se considera que existe una población 31 organizaciones corporativas petroquímicas operando en el estado de Tamaulipas, la lucha por el mercado se vuelve muy agresivo y complicado. Se eligen las organizaciones corporativas petroquímicas, porque las micro, pequeñas y medianas empresas no están en posibilidades de adquirir el almacén de datos.

La rentabilidad es la utilidad o pérdida neta a capital contable (porciento) (Martínez, 2000); es la forma de medir el retorno en utilidades que se tiene con respecto de una inversión determinada. Retorno de la Inversión (ROE) es el margen neto multiplicado por el coeficiente de ingresos entre activos (porciento). Dos hechos resaltan de la realidad de las organizaciones corporativas petroquímicas: la inexistencia de una planeación a largo plazo y la carencia de proyectos ligados a la tecnología dentro de las estrategias corporativas. Ambos factores frenan la influencia en la asimilación exitosa de la tecnología de información de almacén de datos, así como también una adecuada toma de decisión para adquirir la tecnología debe partir de un proceso que debe alinear el 
objetivo de la organización con el almacén de datos de cada una de las áreas del proceso, sea finanzas, ventas, logística o distribución.

\section{MATERIALES Y MÉTODOS}

Hipótesis de este trabajo

E almacén de datos en las organizaciones corporativas petroquímicas disminuye los errrores en la toma de decisiones y se refleja en la rentabilidad. La presente investigación se caracteriza por ser no experimental, por tener un corte de tipo transversal, el tema de estudio es un campo con escasos estudios, se encontró un vacío teórico muy importante de análisis debido a la situación de que las organizaciones corporativas petroquímicas son las que cuentan con almacén de datos, razón para profundizar en el estudio de la relación entre el proceso de asimilación y la rentabilidad.

Se precisaron los indicadores para cada una de las dimensiones, fué el punto de partida para la elaboración de los ítems del cuestionario (Basado en la Tabla 2), siendo la población de treinta y una organizaciones corporativas petroquímicas, formada por quince responsables de los centros de informática y dieciséis ejecutivos responsables de la toma de decisiones. Se aplicó una encuesta, dividida en 5 fases, con sesenta y tres ítems, se describe textualmente la selección de las etapas del diseño del almacén de datos.

\section{Instrumento}

Se emplea un grupo de respuestas de cinco puntos, para obtener las puntuaciones de la escala de Likert, el grado de adopción total es cuatroscientos setenta y cinco, la puntuación se considera alta o baja respecto del puntaje total; este último está dado por el número de ítems o afirmaciones multiplicada por cinco (Tabla 1$)$.

Tabla 1: Proceso de Asimilación del almacén de datos

\begin{tabular}{ccc}
\hline Puntos & $\begin{array}{c}\text { Grado de adopción del almacén } \\
\text { de datos }\end{array}$ \\
\hline 1 & $000-95$ & Muy Bajo \\
2 & $096-190$ & Bajo \\
3 & $191-285$ & Medio \\
4 & $286-380$ & Alto \\
5 & $381-475$ & Muy alto \\
\hline
\end{tabular}

Se resume en la tabla 2 el modelo propuesto, las medidas de evaluación del grado de adopción de cada fase son los atributos: ventaja relativa es el grado con la cual el almacén de datos se percibe como mejor que la idea que sustituye o reemplaza, compatibilidad es el grado con el cual una innovación se descubre por los posibles adoptadores como consistente con sus valores existentes, experiencias pasadas y necesidades, complejidad es el grado con el cual un almacén de datos se aprecia como relativamente difícil para entenderla o utilizarla, experimentabilidad es el grado con el cual una innovación puede experimentarse bajo bases limitadas, observabilidad es el grado con el cual los resultados de un almacén de datos son evidentes a otros.

De manera paralela a la implantación del almacén de datos se propone analizar estos 5 atributos para determinar el grado de adopción que va teniendo el recurso humano durante cada etapa de implantación de la nueva tecnología: ventaja relativa, compatibilidad, complejidad, experimentabilidad y observabilidad, influyen en el proceso y en las etapas de las propuestas de diseño de almacén de datos presentadas, a lo cual pueden agregar nuevos atributos que consideren influyan en la asimilación. Es importante remarcar que la finalidad de aplicar este proceso de asimilación en una organización corporativa petroquímica es lograr que las tecnologías almacén de datos sean aceptadas y asimiladas por el personal que va a utilizar las herramientas del software. 
Tabla 2: Fases del modelo del proceso asimilación del almacén de datos en la organización corporativa petroquímica

\begin{tabular}{|c|c|c|}
\hline \multicolumn{2}{|c|}{ 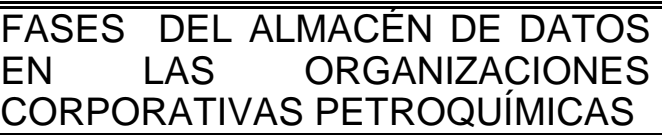 } & ETAPAS \\
\hline I & Investigación & Búsqueda del almacén de datos disponible. \\
\hline II & Conocimiento- decisivo & $\begin{array}{l}\text { Conocimiento del almacén de datos, establecimiento de } \\
\text { las necesidades de la organización corporativa } \\
\text { petroquímica, consideración y decisión de la adopción, } \\
\text { discusión para la adquisición. }\end{array}$ \\
\hline III & Evaluación - Decisión & $\begin{array}{l}\text { Proposición de adquisición del almacén de datos, } \\
\text { evaluación racional-financiera, evaluación política- } \\
\text { estratégica, evaluación de aptitud organizacional, } \\
\text { métricas para evaluar las inversiones en la prueba o } \\
\text { experimentación, decisión de adopción, rechazo o } \\
\text { posposición del uso del almacén de datos. }\end{array}$ \\
\hline IV & Introducción - Adopción & $\begin{array}{l}\text { Redefinición, reestructuración y planeación de la } \\
\text { introducción del almacén de datos, aprendizaje y } \\
\text { clarificación del uso del almacén de datos, difusión y } \\
\text { reconocimiento de logros del uso del almacén de datos, } \\
\text { creación de ontologías decisionales, adopción, } \\
\text { aceptación y rutinización del uso del almacén de datos. }\end{array}$ \\
\hline $\mathrm{V}$ & Confirmación- Difusión & $\begin{array}{l}\text { Evaluación del uso del almacén de datos, confirmación } \\
\text { del uso del almacén, difusión e institucionalización del } \\
\text { uso del almacén de datos, métricas para evaluar las } \\
\text { inversiones y capital humano. }\end{array}$ \\
\hline
\end{tabular}

\section{RESULTADOS Y DISCUSIÓN}

Se resumen los resultados (Fig. 1 y 2), las áreas funcionales deben estar integradas todas en el almacén de datos y compartir la información, para tener toda la confianza en la toma de decisiones al consultarlo en las organizaciones corporativas del estado de Tamaulipas, es por esto que en las fases la puntuación que se asigna es alta y muy alta, en donde aseguran los encuestados que las estrategias de la organización corporativa pueden apoyarse en la información del almacén de datos, en las cinco fases hay un punto fuera del grado de aceptación, que corresponde al ítem $6 \mathrm{E}$ de la fase V, en la medida de evaluación ventaja relativa: ¿Cuál es la capacidad utilizada del almacén de datos en su organización corporativa petroquímica?

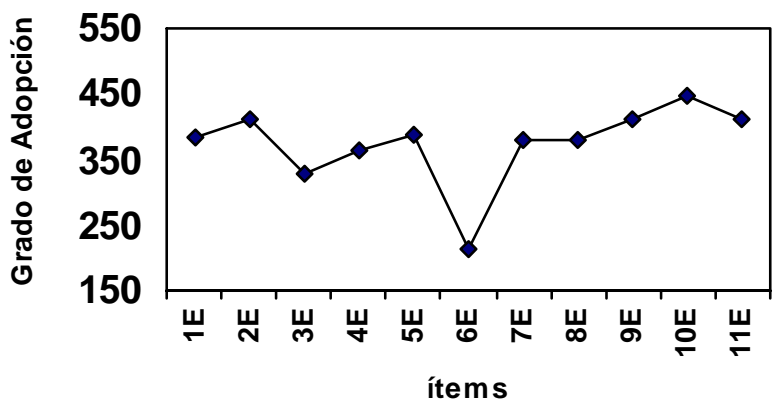

Fig. 1:Fase V Confirmación difusión

En promedio, se utiliza únicamente el $42 \%$ de la capacidad del almacén de datos en las organizaciones corporativas petroquímicas, por lo que no se explotan al máximo sus potencialidades, tiene poco uso de sus capacidades y habilidades, lo que ocasiona pérdidas millonarias a las 
organizaciones corporativas petroquímicas. Los resultados de la encuesta se demuestran estadísticamente a 2.48 desviaciones estándar, se tiene una confianza del $100 \%$, se aprueba la hipótesis ya que podemos apreciar que el grado de adopción promedio es muy alto de 392.93.

Se analizaron los componentes que conforman la arquitectura de información, se puede visualizar que todos persiguen un objetivo común: administrar estratégicamente los datos para mejorar el flujo de la información en la organización corporativa y apoyar de esta manera el proceso de toma de decisiones, en general se consideró que la rentabilidad del proceso de toma de decisiones de la organización corporativa petroquímica, empleando el almacén de datos es del $81.05 \%$, al tener este objetivo, el almacén de datos cumple con la meta de toda arquitectura de información desde un punto de vista tecnológico.

El enfoque de solución funciona el almacén de datos apoya el ahorro financiero en un 86.73\%, haciendo eficiente el flujo de información en la toma de decisiones, para lo cual se buscó relacionar directamente el diseño organizacional con el diseño de la arquitectura del almacén de datos.

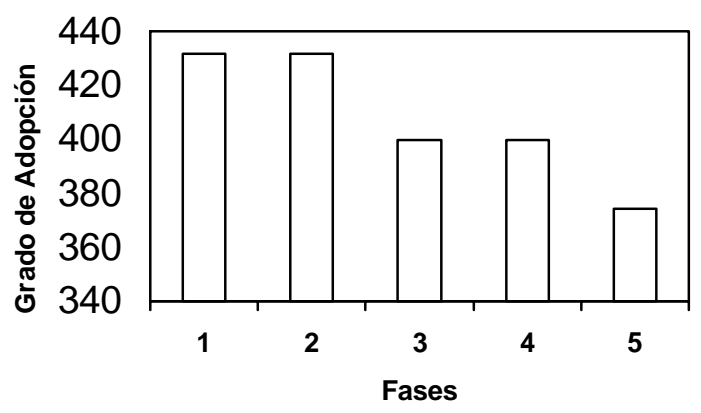

Fig. 2: Grado de adopción de las organizaciones corporativas petroquímicas en el estado de Tamaulipas, México

Actualmente se utiliza el $42 \%$ de la capacidad del almacén de datos sólo se tiene instalado el almacén de datos en una o varias áreas funcionales y en éstas áreas el grado de adopción se encontró que es muy alto, pero en las otras áreas no tienen toda la información en tiempo real de la organización corporativa petroquímica, lo que significa la pérdida de oportunidades que ocasionan gastos, ya que alguien tiene que procesar y entregar los datos que necesitan los responsables de la toma de decisiones, la forma en que presentan o reciben los reportes y/o los informes.

Se encontró que la información tiene poco valor por sí misma en un 91\% y sólo se convierte en conocimiento cuando es procesada por el cerebro humano. El almacén de datos apoya el ahorro financiero en un $89.7 \%$, haciendo eficiente el flujo de información en la toma de decisiones, para lo cual se buscó relacionar directamente el diseño organizacional corporativo petroquímico con el diseño de la arquitectura del almacén de datos. Es necesario que sea un sistema abierto para que se pueda implantar el proceso de asimilación, ya que un sistema cerrado tiene límites rígidos e impenetrables; en tanto que los del sistema abierto son límites permeables y moldeables. El proceso de asimilación del almacén de datos en las organizaciones corporativas petroquímica en el estado de

\section{CONCLUSIONES}

La aportación es una propuesta de modelo enfocada específicamente al proceso de asimilación del almacén de datos en las organizaciones corporativas petroquímicas, lo que se ha hecho antes sirve como base en el desarrollo del modelo, se fundamentó en modelos ya probados, el modelo propuesto es mejor porque se considera que los objetivos de la organización estén alineados al almacén de datos, estableciendo métricas para evaluar el almacén de datos antes y después de adquirilo, fundamentándose en un plan informático para un entorno cambiante, se considera la capacitación, la intervención de agentes de cambio y de expertos. 
El proceso de asimilación del almacén de datos en las organizaciones corporativas petroquímicas en el estado de Tamaulipas, dsiminuyen el tiempo, traduciéndose en un incremento de la prontitud y presición para la toma de decisiones, se refleja en un $93.9 \%$ en la rentabilidad, al tener este objetivo, el almacén de datos cumple con la meta de toda arquitectura de información desde un punto de vista tecnológico.

\section{REFERENCIAS}

Cota, I.E.; "Construcción de un data warehouse", Memorias del Congreso Avances en Tecnologías de la Información CNCIIC 2007, ISBN 878-970-31-0949-4, 327-335 ( 2007).

Giovinazzo, W. e Inmon, B.; "Object-Oriented Data Warehouse Desing: Building a Star Schema”, Ed. Wiley, ISBN: 0-13-085081-0, 96-99 (2001).

Gómez, A.; "Industria TIC: Percepciones de los directivos mexicanos sobre el valor de las tecnologías de información y comunicaciones (TIC), Select en tus decisiones TIC", boletín (2006) http://www.select.com.mx/sec_boletin.asp, acceso: 2 de mayo (2008).

Hernández, F.E.; "Química y Petroquímica, Gobierno del estado de Tamaulipas", 2005 www.tamaulipas.gob.mx/tamaulipas/seconomico/industria/quimico.htm, Acceso: 5 de mayo (2008).

Koncilia C.; "Data Warehouse and OLAP:Concepts, Architectures and Solutions", Imprint: IRM Press, Penn State University, USA, ISBN: 1-59904-364-5, 56-63 (2007).

Lee, Heeseok, Kim, Taehun y Kim, Jongho; "A Metadata Oriented Architecture for Building Data warehouse", Korea Advanced Institute of Science and Technology, Korea. Journal of Database Management: 12(4), 26-27 (2006).

Malori, C; Murguía, S. y Sánchez, R., “Mundo Ejecutivo”: 24 (288), 58-63 (2003).

Mundy, J. Warren T. y Kimball, R.; "The Microsoft Almacén de datos Toolkit: With SQL Server 2005 and the Microsoft Business Intelligence Toolset", Ed.Wiley, ISBN-10: 0471267155, ISBN-13: 9780471267157, 178-181 (2006).

Martínez, S.J.; Las Empresas más Importantes de México, Revista Expansión: ISSN 0185- 2728, 31(795), 49 (2000).

Pérez, C.; "Data Mining: Soluciones con Enterprise Miner". Ed. RA-MA, 22-28 (2006).

Serrano, M.A.; "Empirical studies to assess the understandability of data warehouse schemas using structural metrics". Software Quality Journal: 16 (1), 79-106 (2008).

Sturm, J.; “Data Warehousing with Microsoftr SQL Server ${ }^{\mathrm{TM}}$ 7.0 Technical Reference”, ISBN 0-73560859-8., Editorial: Redmond, Washington, 121- 126 (2006). 